High frequency performance of individual and arrays of single-walled carbon nanotubes

This content has been downloaded from IOPscience. Please scroll down to see the full text.

2012 Nanotechnology 23245202

(http://iopscience.iop.org/0957-4484/23/24/245202)

View the table of contents for this issue, or go to the journal homepage for more

Download details:

IP Address: 139.179.2.250

This content was downloaded on 23/05/2014 at 07:08

Please note that terms and conditions apply. 


\title{
High frequency performance of individual and arrays of single-walled carbon nanotubes
}

\author{
Osman Balci and Coskun Kocabas \\ Advanced Research Laboratories, Department of Physics, Bilkent University, 06800 Ankara, Turkey \\ E-mail: ckocabas@fen.bilkent.edu.tr
}

Received 5 February 2012, in final form 14 April 2012

Published 28 May 2012

Online at stacks.iop.org/Nano/23/245202

\begin{abstract}
We have studied the high frequency performance limits of single-walled carbon nanotube (SWNT) transistors in the diffusive transport regime limited by the acoustic phonon scattering. The relativistic band structure of single-walled carbon nanotubes combined with the acoustic phonon scattering provides an analytical model for the charge transport of the radio frequency transistors. We were able to obtain the intrinsic high frequency performance such as the cut-off frequency and the linearity of the SWNT transistors. We have extended our model to include transistors based on arrays of SWNTs. The effect of electrostatic screening in a dense array of SWNTs on the cut-off frequency is studied.
\end{abstract}

(Some figures may appear in colour only in the online journal)

Low-dimensional carbon, such as single-walled carbon nanotubes (SWNTs) [1-4], graphene [5, 6] and hydrogen passivated diamond surface $[7,8]$ has remarkable electronic properties for high frequency electronics. These unusual electronic properties originate from the unique crystal and electronic band structure which provides extremely high carrier mobilities $[9,10]$. Owing to the high charge mobility of SWNTs and low geometric capacitance, carriers on the tubes can respond to an external electric field in a picosecond time scale [11]. This very fast response time opens new prospects for high frequency device applications. Carbon nanotube transistors for radio frequency (RF) electronics have been the subject of intensive research for the last decade [1, 12, 2, 3, 13]. SWNT based RF transistors [2, 14, 15], oscillators [16, 17] and analog electronic circuits [18, 3] have been demonstrated. The main practical limitation of nanotube transistors for high frequency operation is the parasitic fringing capacitance of the contact electrodes. The electrostatic capacitance of an SWNT is around $100 \mathrm{aF} \mu \mathrm{m}^{-1}$ [19], which is much smaller than the fringe capacitance between the gate and source/drain electrodes which is larger than $500 \mathrm{aF} \mu \mathrm{m}^{-1}$ [2]. These drawbacks make single-tube devices impractical for high frequency electronics. Dense arrays of SWNTs, however, increase the total gate capacitance and the contribution of the fringe capacitance becomes smaller. More recent studies show that a dense array of SWNTs provides a more reliable platform for fabrication and characterization of RF transistors and circuits [2, 3, 20-22].

There have been many theoretical and experimental studies to understand the ultimate limit of the performance of SWNTs in the radio frequency band [12-14, 23-25]. However, there has been no study to understand the intrinsic effect of the electronic band structure on high frequency operation. In this work, we study the ultimate performance limit of SWNT transistors operating in the radio frequency band. Firstly, we analyze the intrinsic performance of a transistor based on a single semiconducting tube with a relativistic like band structure [26, 9] coupled with the acoustic phonon scattering [27]. After the analysis of single-tube devices we then extend our model to study dense arrays of SWNTs. The model device used for the calculation is a top-gated transistor based on a recent experimental study of an SWNT RF transistor [2]. For the model, we have used realistic experimental parameters such as $50 \mathrm{~nm}$ thick $\mathrm{HfO}_{2}$ as the gate dielectric and a channel length of $700 \mathrm{~nm}$. Figures 1(a) and (b) show schematic representations of a 
(a)

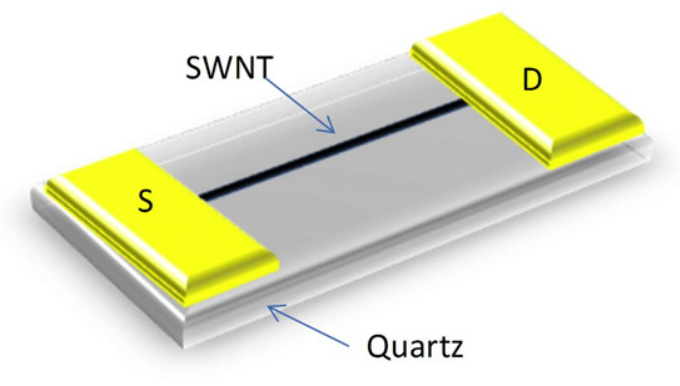

(c)

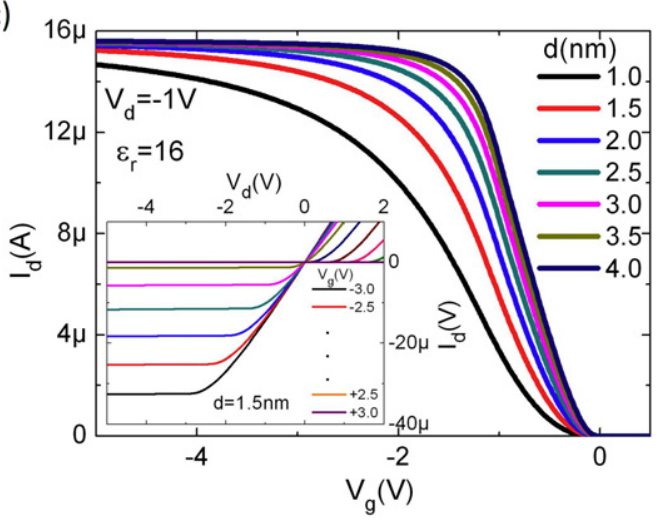

(b)

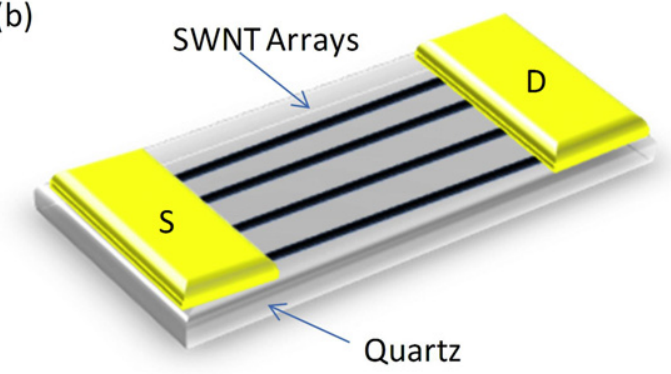

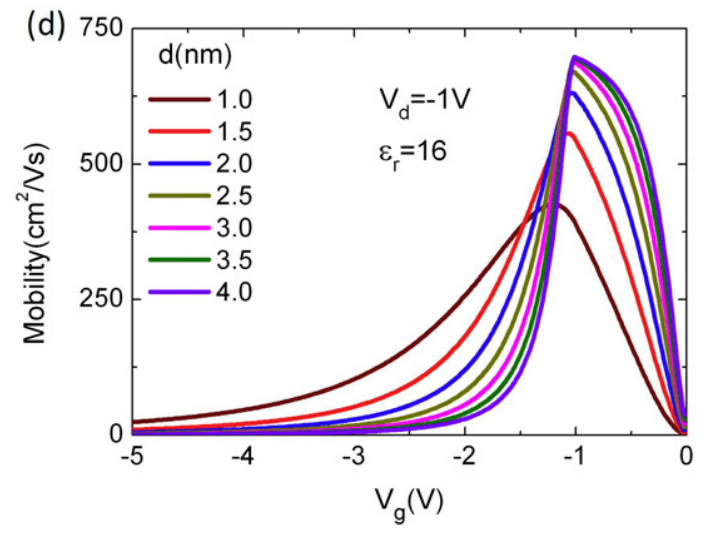

Figure 1. Schematic representation of a model transistor based on (a) a single SWNT and (b) arrays of SWNTs. The gate electrode and dielectric are not shown in the figure. (c) Calculated transfer characteristics of single-tube transistors using the relativistic band structure for a range of diameters between 1 and $4 \mathrm{~nm}$. The inset shows the calculated output characteristics of a device with a tube diameter $1.5 \mathrm{~nm}$. The parameters used for the calculations are a channel length of $700 \mathrm{~nm}$, a gate dielectric thickness of $50 \mathrm{~nm}$ and a drain voltage of $-1 \mathrm{~V}$.

(d) The calculated field effect mobility as a function of gate voltage for tube diameters of between 1 and $4 \mathrm{~nm}$.

transistor based on a single SWNT and arrays of SWNTs respectively.

For the diffusive transport regime the conductance of a 1D conductor with four conduction channels is given as $G=\frac{4 e^{2}}{h} \frac{l_{\mathrm{f}}}{L_{\mathrm{c}}}$ where $l_{\mathrm{f}}$ is the mean free path, $L_{\mathrm{c}}$ is the channel length. The conductivity along the tube (at a point $x)$ can be written as $\sigma(x)=\frac{4 e^{2}}{h} l(x)$ where $l(x)$ is the mean free path along the channel. For $1 \mathrm{D}$, the scattering rate is proportional to the density of states [28, 29], therefore the mean free path along the channel can be written as $l(x)=$ $l_{0}\left(\frac{v(x)}{v_{0}}\right)^{2}$ where $v(x)$ and $v_{0}$ are the Fermi velocity of the SWNT along the channel and at high energies, respectively. The mean free path at high energies is written as $l_{0}=$ $v_{0} \tau_{0}$ where $\tau_{0}^{-1}$ is the total scattering rate. To estimate the scattering time, we should consider the length scale and energies of acoustic and optical phonons. The experimentally observed mean free paths at low and high bias conditions are $1.6 \mu \mathrm{m}$ and $10 \mathrm{~nm}$, respectively [30]. Acoustic phonons have energies less than the thermal energy $k_{\mathrm{b}} T(T=300 \mathrm{~K})$. Optical phonons have energies $(\sim 0.2 \mathrm{eV})$ much larger than the room temperature thermal energy. Therefore at room temperature and low bias conditions the scattering rate is dominated by acoustic phonons as $\tau_{0}^{-1}=\alpha \frac{T}{d}$ where $T$ is the temperature and $d$ is the diameter of the tube [31]. The measured value for $\alpha$ is $12 \mathrm{~m} \mathrm{~K}^{-1} \mathrm{~s}^{-1}$ [30]. For the calculation we used room temperature values of the mean free path for semiconducting tubes. The Fermi velocity along the channel for a semiconducting tube can be derived from the relativistic band structure $\left(E= \pm \sqrt{\left(m^{*} v_{0}^{2}\right)^{2}+\left(\hbar k v_{0}\right)^{2}}\right)$ of the tubes as [9]

$$
\left(\frac{v(x)}{v_{0}}\right)^{2}=\frac{\left(\frac{h k}{m^{*} v_{0}}\right)^{2}}{1+\left(\frac{h k}{m^{*} v_{0}}\right)^{2}} .
$$

Here $m^{*}$ is the effective mass and $k$ is the wavevector of the carriers in the SWNT. The wavevector depends on the local carrier density and can be written as

$$
k(x)=\frac{\pi}{4} \frac{C_{\mathrm{g}}}{e}\left(V_{\mathrm{g}}-V(x)\right) .
$$

Here $V_{\mathrm{g}}$ is the gate voltage and $V(x)$ is the local potential along the tube, $C_{\mathrm{g}}$ is the total capacitance between the tube and the gate electrode. The total capacitance $C_{\mathrm{g}}$ includes both the electrostatic capacitance $C_{\mathrm{e}}$ and the quantum capacitance $C_{\mathrm{q}}$ in series $\left(C_{\mathrm{g}}^{-1}=C_{\mathrm{e}}^{-1}+C_{\mathrm{q}}^{-1}\right)$. We can write the quantum capacitance in terms of the electronic density of states $g(\varepsilon)$ and the velocity $v(x)$ as $C_{\mathrm{q}}=e^{2} g(\varepsilon)=\frac{8 e^{2}}{h v(x)}$. Using equation (1), we can write $C_{\mathrm{q}}$ as

$$
C_{\mathrm{q}}=\frac{16 e^{2} m^{*}}{h^{2} k} \sqrt{1+\left(\frac{h k}{2 \pi m^{*} v_{0}}\right)^{2}} .
$$



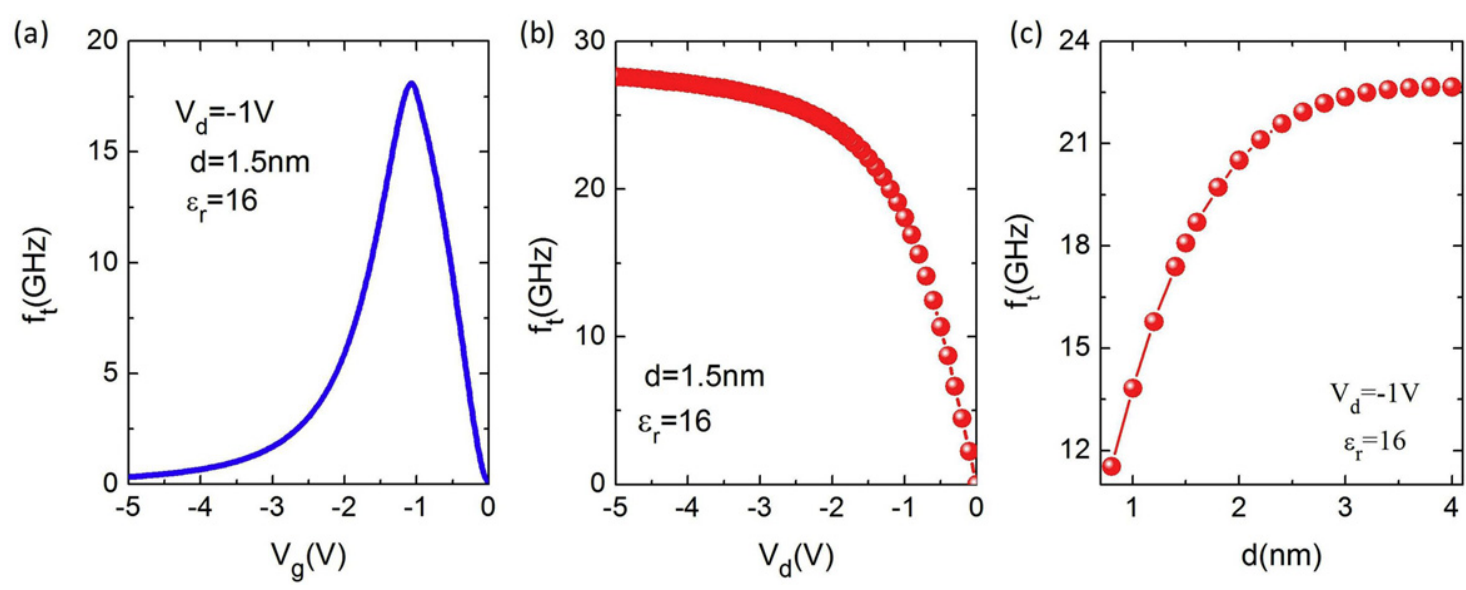

Figure 2. The calculated high frequency performance of an SWNT transistor based on a single tube with a diameter of $1.5 \mathrm{~nm}$. (a) Gate voltage dependence of the calculated cut-off frequency $\left(f_{\mathrm{t}}\right)$. The peak cut-off frequency and the peak mobility scale similarly. (b) Drain bias dependence of the cut-off frequency. (c) Scaling of the peak cut-off frequency with the tube diameter. The intrinsic $f_{\mathrm{t}}$ saturates around $23 \mathrm{GHz}$ for diameters larger than $3 \mathrm{~nm}$.

After calculating the capacitance self-consistently from equations (2) and (3), we write the drain current for a 1D field effect transistor as

$$
I_{\mathrm{d}}=\frac{1}{L_{\mathrm{c}}} \int_{0}^{L_{\mathrm{c}}} \sigma(x) E(x) \mathrm{d} x=\frac{1}{L_{\mathrm{c}}} \int_{V_{\mathrm{s}}}^{V_{\mathrm{d}}} \sigma(V) \mathrm{d} V
$$

where $\sigma$ is the conductivity, and $V_{\mathrm{s}}$ and $V_{\mathrm{d}}$ are the source and drain voltages, respectively. The solution of equation (4) as a function of bias voltages provides the electrical characteristics of the device.

Figure 1(c) shows the calculated transfer curves of SWNTs with diameters from 1.0 to $4.0 \mathrm{~nm}$. The inset shows the output characteristics of an SWNT with a diameter of $1.5 \mathrm{~nm}$. The clear current saturation is seen at negative voltages due to p-type conduction. Owing to the large work function of the SWNTs around $4 \mathrm{eV}$, gold and palladium electrodes provide hole type conduction. For the model we only include hole conduction. The calculated gate voltage dependence on the field effect mobility of the tube ( $\mu=$ $\left.\frac{\mathrm{d} I_{\mathrm{d}}}{\mathrm{d} V_{\mathrm{g}}} \frac{L_{\mathrm{c}}}{V_{\mathrm{d}} C_{\mathrm{g}}}\right)$ is given in figure $1(\mathrm{~d})$ for a range of tube diameters. The calculated peak field effect mobility is around $700 \mathrm{~cm}^{2} \mathrm{~V}^{-1} \mathrm{~s}^{-1}$. Tubes with smaller diameters show lower peak mobilities. These values agree well with the experimental results [2].

The cut-off frequency $f_{\mathrm{t}}$ of an FET is defined as the frequency where the current gain is zero. This means that for frequencies higher than $f_{\mathrm{t}}$, the gate leakage current is larger than the drain current due to the modulation of the channel resistance. A simplified expression for $f_{\mathrm{t}}$ is

$$
f_{\mathrm{t}}=\frac{g_{m}}{2 \pi C_{\mathrm{g}}}
$$

where $g_{m}$ is the transconductance of the device. The performance of the SWNT FET is limited by the total gate capacitance. To understand the intrinsic performance we only consider the channel capacitance excluding the parasitic capacitances. Figure 2(a) shows the gate voltage dependence of the calculated cut-off frequency of the device given in figure 1 (c) with $d=1.5 \mathrm{~nm}$. The $f_{\mathrm{t}}$ reaches a maximum value at the gate voltage which provides the maximum transconductance. The drain voltage dependence of $f_{\mathrm{t}}$ of the same device is given in figure 2(b). Due to the current saturation, $f_{\mathrm{t}}$ saturates at large drain voltages. A useful piece of information would be to see the diameter dependence of the cut-off frequency. The diameter of a tube affects the transconductance and capacitance of the device in opposite ways. Figure 2(c) shows the calculated diameter dependence of the intrinsic cut-off frequency. The cut-off frequency increases with tube diameter and saturates after $3 \mathrm{~nm}$. The saturation value of the cut-off frequency is around the intrinsic cut-off frequency of graphene [32].

Dense arrays of SWNTs provide a more practical configuration for high frequency operation than a single tube. A significant piece of information can be obtained by studying the intrinsic performance of an individual SWNT in a dense array. Increasing the density of the tubes decreases the contribution of fringing capacitance and allows us to achieve the intrinsic performance of the SWNTs. Electrostatic screening of the tubes in an array configuration also affects the performance of the device. Figure 3(a) shows a cross sectional view of an FET based on SWNT arrays. The thickness of the gate dielectric, $t$, and the distance between tubes, $L$, will define the effect of the screening. When the tubes are closer than the thickness of the gate dielectric, the tubes start to screen each other and result in reduction of the electrostatic capacitance of an individual tube in the array. The electrostatic capacitance of a single tube with a radius of $r$ in an array with a density of $D$ is given as $[33,34]$

$$
C_{\text {array }}=\frac{2 \pi \varepsilon_{o} \varepsilon_{r}}{\ln \left[\sinh \left(\frac{\pi(2 t+r)}{L}\right) / \sinh \left(\frac{\pi r}{L}\right)\right]}
$$

where $L$ is the average distance between the tubes and $t$ is the thickness of the gate dielectric. This expression is an approximation assuming that the charge distribution on the tube is symmetric [35]. Figure 3(b) shows the calculated electrostatic capacitances of individual tubes in 
(a)

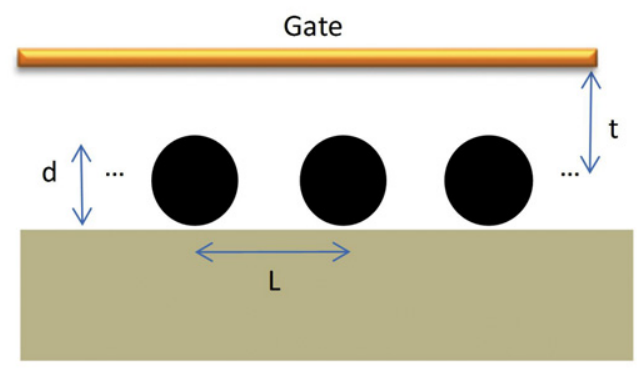

(c) $16 \mu$

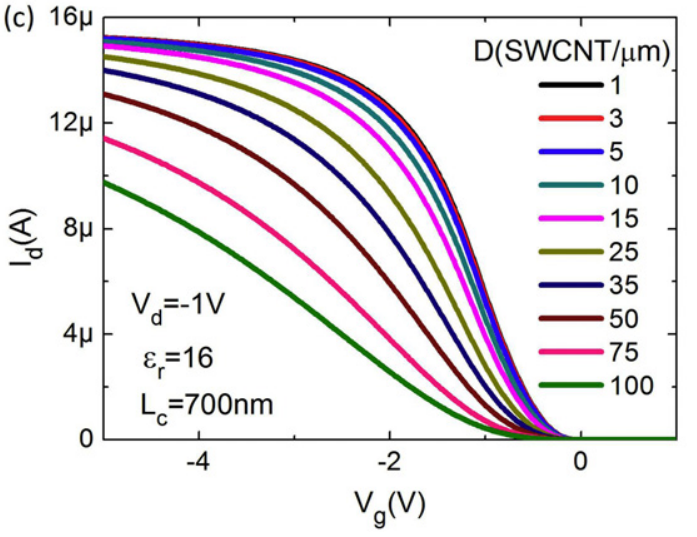

(b) $160 p$

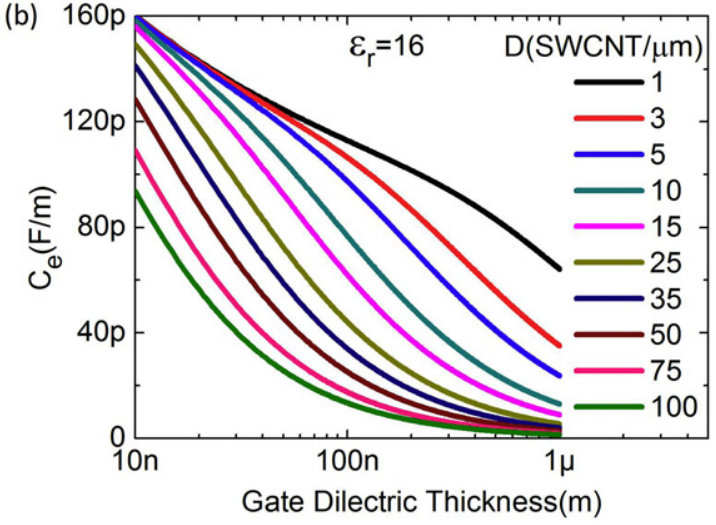

(d)

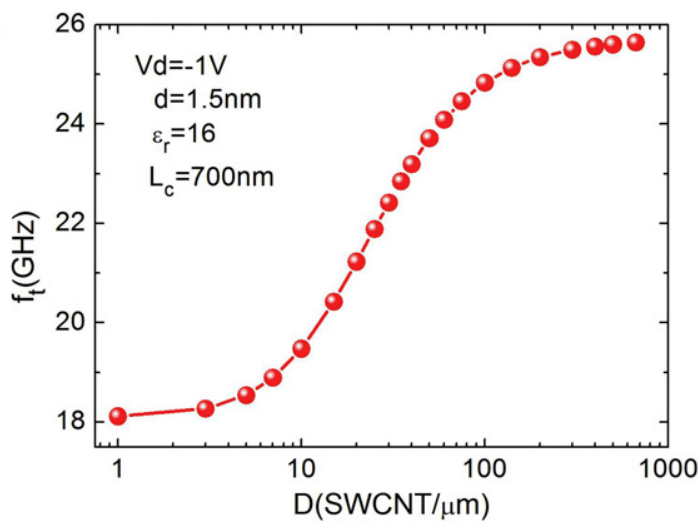

Figure 3. High frequency performance of SWNT arrays. (a) Schematic illustration of the geometry used for the calculation of the electrostatic gate capacitances of SWNT arrays. $d$ is the diameter of the tubes, $L$ is the separation between the tubes and $t$ is the thickness of the gate dielectric. (b) Calculated capacitances of SWNT arrays with tube densities between 1 and $100 \mathrm{SWNT}_{\mu \mathrm{m}^{-1}}$. (c) Calculated transfer curves of single nanotubes in arrays with tube densities between 1 and $100 \mathrm{SWNT} \mu \mathrm{m}^{-1}$. Here the average tube diameter used is $1.5 \mathrm{~nm}$. (d) Calculated intrinsic cut-off frequency of an SWNT array as a function of tube density. The cut-off frequency saturates for tube densities greater than $\sim 100 \mathrm{SWNT} \mu \mathrm{m}^{-1}$ at $26 \mathrm{GHz}$.

arrays with different tube densities. For sparse arrays $(L \gg t)$ the capacitance approaches the single-tube capacitance $(C=$ $\left.\frac{2 \pi \varepsilon_{o} \varepsilon_{r}}{\ln \left(\frac{2 t r}{r}\right)}\right)$ and, on the other hand, for large densities $(L \ll t)$ the capacitance approaches the parallel plate capacitance. Including the effect of electrostatic screening in the array configuration, we calculated the transfer curve for an individual SWNT as a function of tube density. For this calculation we followed the same procedure as we used for an individual tube. As the density approaches 100 tube $\mu \mathrm{m}^{-1}$ there is a significant reduction in the transconductance. The tube density affects the cut-off frequency by decreasing the transconductance and the gate capacitance. Figure 3(d) shows the density dependence of the cut-off frequency of a device based on SWNT arrays. As the tube density increases the capacitance decreases faster than the transconductance resulting in an increase in the cut-off frequency. An SWNT with a diameter of $1.5 \mathrm{~nm}$ in an array configuration provides $f_{\mathrm{t}}$ values of $18 \mathrm{GHz}$ and $25 \mathrm{GHz}$ for 1 and 100 SWNT tube densities, respectively. The thickness of the gate dielectric $(t)$ and the average distance between tubes $(L)$ define important length scales. Up to a tube density of 10 tube $\mu \mathrm{m}^{-1}$ (where $L=100 \mathrm{~nm}$ and $t=50 \mathrm{~nm}$ ) there is a slight effect of screening in the array. Experimentally, synthesis of SWNT arrays with tube densities of more than 10 tube $\mu \mathrm{m}^{-1}$ still remains a challenge $[34,36]$. In order to avoid screening effects, the most suitable configuration is to use a dielectric with a thickness less than the average tube separation.

Another important figure of merit for high frequency operation is the linearity of the device $[37,38]$. The linearity of a transistor is defined as ratio of the first and third derivatives of the transfer curve. This is called the third order intercept $\left(\mathrm{IP} 3=\left(\frac{4}{3}\right) g_{m} / g_{m}^{\prime \prime}\right)$. It has been predicted that ohmically contacted, ballistic nanotube transistors with large electrostatic capacitance (larger than the quantum capacitance) are inherently linear [37]. The ratio of electrostatic to quantum capacitance $\left(C_{\mathrm{e}} / C_{\mathrm{q}}\right)$ defines the inherent linearity. Using our model we studied the effect of band structure on the linearity of SWNT transistors. Figure 4(a) shows the transfer curves for a transistor calculated for a wide range of $C_{\mathrm{e}} / C_{\mathrm{q}}$ ratios. As the electrostatic capacitance increases, the transfer curve becomes more linear. The calculated normalized IP3 of the device as a function of gate voltage is given in figure 4(b). We observe that the linearity of an SWNT transistor depends on diameter of the tube. Figure 4(c) shows the calculated maximum IP3 as a function of electrostatic capacitance for a range of tube diameters. As shown in the inset of figure 4(c), increasing the tube diameter will further increase the linearity of the SWNT 


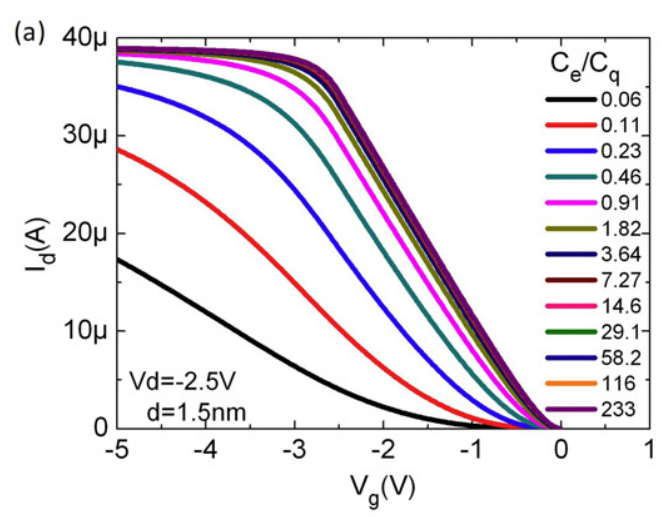

(c)

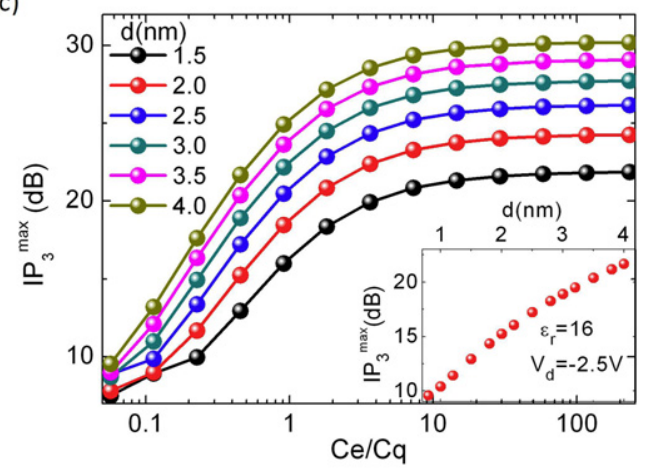

(b)

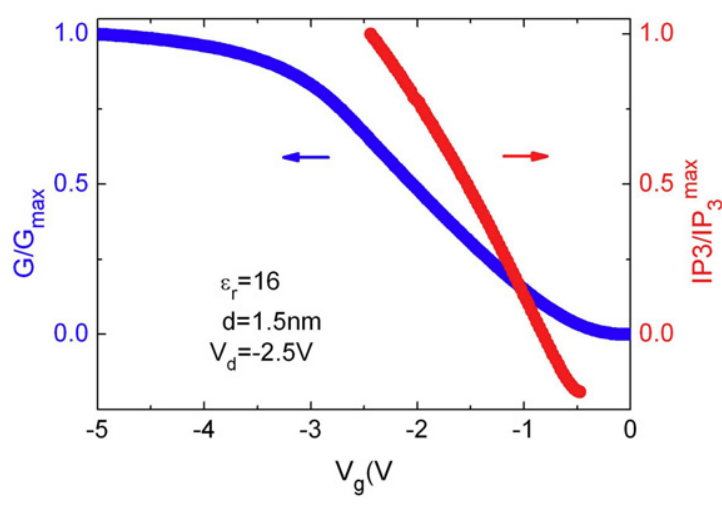

(d)

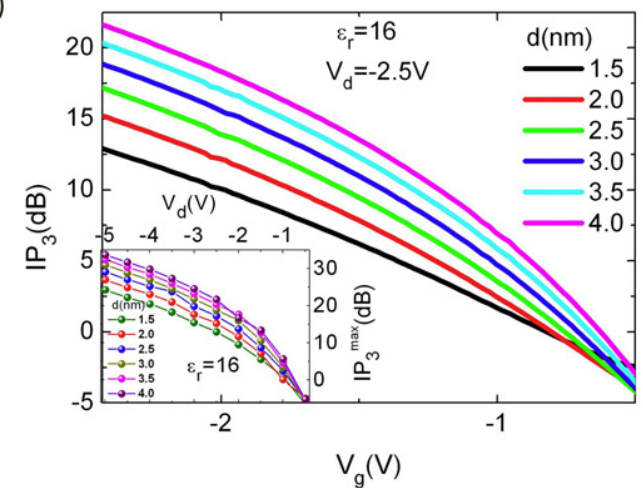

Figure 4. The linearity of SWNT FETs. (a) Transfer curves of a transistor calculated for several values of the ratio of electrostatic to quantum capacitance $\left(C_{\mathrm{e}} / C_{\mathrm{q}}\right)$ at a drain voltage of $-2.5 \mathrm{~V}$. The transfer curve becomes more linear as the ratio increases. (b) The calculated normalized conductance and the third order intercept (IP3) as a function of gate voltage. (c) Maximum values of IP3 for tube diameters between 1.5 and $4.0 \mathrm{~nm}$ as a function of the $C_{\mathrm{e}} / C_{\mathrm{q}}$ ratio. The inset shows the scaling of the IP3 values with the tube diameter. Here the drain voltage is $-2.5 \mathrm{~V}$ and $\varepsilon_{r}=16$ where $C_{\mathrm{e}} / C_{\mathrm{q}}$ is equal to 0.46 . (d) Maximum IP3 as a function of the gate and drain (inset) voltages for diameters from 1.5 to $4.0 \mathrm{~nm}$.

transistor. The bias voltage dependence of IP3 is shown in figure 4(d). We anticipate that in the large diameter limit, the linearity value of an SWNT device will approach that of a graphene transistor with a similar device geometry [39].

In summary we have studied the ultimate performance limit of SWNTs based on a simplified relativistic like band structure coupled with acoustic phonon scattering. The intrinsic high frequency performances of devices based on individual SWNTs and arrays of SWNTs have been characterized. The cut-off frequency of an SWNT increases with the tube diameter and saturates after $3 \mathrm{~nm}$. For the array geometry the intrinsic cut-off frequency increases with tube density and saturates for tube densities around 100 tube $\mu \mathrm{m}^{-1}$. Using the developed model we were also able to calculate the linearities of SWNT transistors. These results reveal the basic intrinsic limitations of high frequency performance of individual SWNTs and arrays of SWNTs and provide a framework to understand the design considerations for high frequency operation.

\section{Acknowledgments}

This work was supported by the Scientific and Technological Research Council of Turkey (TUBITAK) grant no. 109T259, Marie Curie International Research Grant (IRG) grant no. 256458 and the Turkish Academy of Science (TUBAGEBIP).

\section{References}

[1] Burke P J 2004 AC performance of nanoelectronics: towards a ballistic THz nanotube transistor Solid-State Electron. 48 1981-6

[2] Kocabas C et al 2009 High-frequency performance of submicrometer transistors that use aligned arrays of single-walled carbon nanotubes Nano Lett. 9 1937-43

[3] Kocabas C, Kim H S, Banks T, Rogers J A, Pesetski A A, Baumgardner J E, Krishnaswamy S V and Zhang H 2008 Radio frequency analog electronics basedon carbon nanotube transistors Proc. Natl Acad. Sci. USA 105 1405-9

[4] Rutherglen C, Jain D and Burke P 2009 Nanotube electronics forradio frequency applications Nature Nanotechnol. 4 811-9

[5] Lin Y M, Dimitrakopoulos C, Jenkins K A, Farmer D B, Chiu H Y, Grill A and Avouris P $2010100 \mathrm{GHz}$ transistors from wafer-scale epitaxial graphene Science 327662

[6] Xia F N, Mueller T, Lin Y M, Valdes-Garcia A and Avouris P 2009 Ultrafast graphene photodetector Nature Nanotechnol. 4 839-43

[7] Kasu M, Ueda K, Ye H, Yamauchi Y, Sasaki S and Makimoto T 2006 High RF output power for H-terminated diamond FETs Diamond Relat. Mater. 15 783-6

[8] Ueda K, Kasu M, Yamauchi Y, Makimoto T, Schwitters M, Twitchen D J, Scarsbrook G A and Coe S E 2006 Diamond 
FET using high-quality polycrystalline diamond with $\mathrm{f}(\mathrm{T})$ of $45 \mathrm{GHz}$ and $\mathrm{f}(\max )$ of $120 \mathrm{GHz}$ IEEE Electron Device Lett. 27 570-2

[9] Zhou X J, Park J Y, Huang S M, Liu J and McEuen P L 2005 Band structure, phonon scattering, and the performance limit of single-walled carbonnanotube transistors Phys. Rev. Lett. 95146805

[10] Jimenez D, Cartoixa X, Miranda E, Sune J, Chaves F A and Roche S 2007 Asimple drain current model for Schottky-barrier carbon nanotube field effecttransistors Nanotechnology 18025201

[11] Zhong Z H, Gabor N M, Sharping J E, Gaeta A L and McEuen P L 2008 Terahertz time-domain measurement of ballistic electron resonance in a single-walled carbon nanotube Nature Nanotechnol. 3 201-5

[12] Frank D J and Appenzeller J 2004 High-frequency response in carbon nanotube field-effect transistors IEEE Electron Device Lett. 25 34-6

[13] Nougaret L, Dambrine G, Lepilliet S, Happy H, Chimot N, Derycke V and Bourgoin J P 2010 Gigahertz characterization of a single carbon nanotube Appl. Phys. Lett. 96042109

[14] Rosenblatt S, Lin H, Sazonova V, Tiwari S and McEuen P L 2005 Mixing at $50 \mathrm{GHz}$ using a single-walled carbon nanotube transistor Appl. Phys. Lett. 87153111

[15] Li S D, Yu Z, Yen S F, Tang W C and Burke P J 2004 Carbon nanotubetransistor operation at 2.6 GHz Nano Lett. 4 753-6

[16] Pesetski A A, Baumgardner J E, Krishnaswamy S V, Zhang H, Adam J D, Kocabas C, Banks T and Rogers J A 2008 A $500 \mathrm{MHz}$ carbon nanotube transistor oscillator Appl. Phys. Lett. 93123506

[17] Rutherglen C and Burke P 2007 Carbon nanotube radio Nano Lett. 7 3296-9

[18] Jensen K, Weldon J, Garcia H and Zettl A 2007 Nanotube radio Nano Lett. 7 3508-11

[19] John D L, Castro L C and Pulfrey D L 2004 Quantum capacitance innanoscale device modeling J. Appl. Phys. 96 5180-4

[20] Ryu K, Badmaev A, Wang C, Lin A, Patil N, Gomez L, Kumar A, Mitra S, Wong H S P and Zhou C W 2009 CMOS-analogous wafer-scale nanotube-on-insulator approach for submicrometer devices and integrated circuits using aligned nanotubes Nano Lett. 9 189-97

[21] Ryu K M, Badmaev A, Gomez L, Ishikawa F, Lei B and Zhou C W 2007 Synthesis of aligned single-walled nanotubes using catalysts defined by nanosphere lithography J. Am. Chem. Soc. 12910104

[22] Kang S J, Kocabas C, Ozel T, Shim M, Pimparkar N, Alam M A, Rotkin S V and Rogers J A 2007 High-performance electronics using dense, perfectly aligned arrays of single-walled carbon nanotubes Nature Nanotechnol. 2 230-6

[23] Akinwande D, Liang J, Chong S, Nishi Y and Wong H S P 2008 Analytical ballistic theory of carbon nanotube transistors: experimental validation, device physics, parameter extraction, and performance projection J. Appl. Phys. 104124514

[24] Alam K and Lake R K 2006 Dielectric scaling of a zero-Schottky-barrier, $5 \mathrm{~nm}$ gate, carbon nanotube transistor with source/drain underlaps J. Appl. Phys. 100024317

[25] Mencarelli D, Rozzi T, Camilloni C, Maccari L, Di Donato A and Pierantoni L 2008 Modelling of multi-wall CNT devices by self-consistentanalysis of multichannel transport Nanotechnology 19165202

[26] Chen Y-F and Fuhrer M S 2005 Electric-field-dependent charge-carrier velocity in semiconducting carbon nanotubes Phys. Rev. Lett. 95236803

[27] Perebeinos V and Avouris P 2010 Inelastic scattering and currentsaturation in graphene Phys. Rev. B 81195442

[28] Datta S 1995 Electronic Transport in Mesoscopic Systems (London: Cambridge University Press)

[29] Guo J 2005 A quantum-mechanical treatment of phonon scattering in carbon nanotube transistors J. Appl. Phys. 98063519

[30] Park J Y, Rosenblatt S, Yaish Y, Sazonova V, Ustunel H, Braig S, AriasT A, Brouwer P W and McEuen P L 2004 Electron-phonon scattering in metallic single-walled carbon nanotubes Nano Lett. 4 517-20

[31] Roche S, Jiang J, Torres L E F F and Saito R 2007 Charge transport in carbon nanotubes: quantum effects of electron-phonon coupling J. Phys.: Condens. Matter 19183203

[32] Pince E and Kocabas C 2010 Investigation of high frequency performance limit of graphene field effect transistors Appl. Phys. Lett. 97173106

[33] Cao Q, Xia M G, Kocabas C, Shim M, Rogers J A and Rotkin S V 2007 Gate capacitance coupling of singled-walled carbon nanotube thin-film transistors Appl. Phys. Lett. 90023516

[34] Kocabas C, Kang S J, Ozel T, Shim M and Rogers J A 2007 Improved synthesis of aligned arrays of single-walled carbon nanotubes and their implementation in thin film type transistors J. Phys. Chem. C 111 17879-86

[35] Lamalle P U 1997 Capacitance of an array of thick rods parallel to the ground-application to the ion cyclotron resonance frequency antenna of the Joint European Torus J. Phys. D: Appl. Phys. 30 78-83

[36] Yuan D N, Ding L, Chu H B, Feng Y Y, McNicholas T P and Liu J 2008 Horizontally aligned single-walled carbon nanotube on quartz from a large variety of metal catalysts Nano Lett. 8 2576-9

[37] Baumgardner J E, Pesetski A A, Murduck J M, Przybysz J X, Adam J D and Zhang H 2007 Inherent linearity in carbon nanotube field-effect transistors Appl. Phys. Lett. 91052107

[38] Wang C, Badmaev A, Jooyaie A, Bao M Q, Wang K L, Galatsis K and Zhou C W 2011 Radio frequency and linearity performance of transistors using high-purity semiconducting carbon nanotubes Acs Nano 5 4169-76

[39] Parrish K N and Akinwande D 2011 Impact of contact resistance on thetransconductance and linearity of graphene transistors Appl. Phys. Lett. $\mathbf{9 8} 183505$ 